\title{
Furanodiene Induces Endoplasmic Reticulum Stress and Presents Antiproliferative Activities in Lung Cancer Cells
}

\author{
Wen-Shan Xu, Yuan-Ye Dang, Jia-Jie Guo, Guo-Sheng Wu, Jin-Jian Lu, \\ Xiu-Ping Chen, and Yi-Tao Wang
} State Key Laboratory of Quality Research in Chinese Medicine and Institute of Chinese Medical Sciences, University of Macau,
Avenue Padre Toma's Pereira S.J., Taipa, Macau 999078, China

Correspondence should be addressed to Jin-Jian Lu, jinjian.lu@163.com and Yi-Tao Wang, ytwang@umac.mo

Received 3 April 2012; Revised 21 June 2012; Accepted 29 June 2012

Academic Editor: Youn Chul Kim

Copyright (๑) 2012 Wen-Shan Xu et al. This is an open access article distributed under the Creative Commons Attribution License, which permits unrestricted use, distribution, and reproduction in any medium, provided the original work is properly cited.

\begin{abstract}
Furanodiene (FUR) is a natural terpenoid isolated from Curcumae Rhizoma, a well-known Chinese medicinal herb that presents antiproliferation activities in several cancer cell lines. In this study, we demonstrated that FUR concentration dependently inhibits the cell proliferation of A549, NIH-H1299, and 95-D lung cancer cells. $\beta$-elemene, another terpenoid isolated from Curcumae Rhizoma, exhibited weaker antiproliferative effects in A549 and NIH-H1299 cells and activities similar to FUR in 95-D cells. FUR significantly inhibited colony formation in A549 and 95-D cells and upregulated both the mRNA and protein expression levels of binding immunoglobulin protein (BIP) and C/EBP homologous protein (CHOP), indicating that endoplasmic reticulum (ER) stress is induced. FUR treatment led to the accumulation of CHOP in the nucleus, which further confirms induction of ER stress. Furthermore, combined treatment of FUR with paclitaxel showed significant synergetic activities in NIH-H1299 and 95-D cells, suggesting its potential roles in combination therapy. These findings provide a basis for the further study of the anticancer effects in vivo and the internal mechanisms of FUR.
\end{abstract}

\section{Introduction}

Lung cancer is a leading type of cancer worldwide and no effective chemotherapy agents for it have yet been developed [1]. Determination of anticancer compounds from traditional herbs may offer new options for lung cancer treatment [2-6]. Curcumae Rhizoma (or Ezhu in Chinese), a commonly used traditional Chinese medicinal herb, has displayed wide and diverse medicinal value for almost one thousand years, including removal of blood stasis and pain alleviation. The herb has been widely prescribed to treat cardiovascular diseases and cancer in Chinese clinical practice [7]. Besides, this herb is also included in some of the traditional Chinese medicine compound recipe, such as compound recipe Ezhu, for cancer treatment. Furanodiene (FUR, Figure 1(a)), a sesquiterpene isolated from Curcumae Rhizoma, inhibits the growth of several human cancer cell lines, including leukemia HL-60 cells, cervical cancer HeLa cells, prostatic cancer PC3 cells, larynx carcinoma Hep-2 cells, gastric carcinoma SGC-7901 cells, and fibrosarcoma HT-1080 cells and also inhibits the growth of uterine cervical and sarcoma 180 tumors in mice $[8,9]$. However, no reports have yet been found on its anti-cancer effects in lung cancer cells and limited studies on its anti-cancer mechanisms are available. The current consensus is that FUR activates p38 mitogenactivated protein kinase and inactivates extracellular signalregulated kinase signaling, inducing cell cycle arrest and caspase-dependent apoptosis, and so on $[9,10]$.

Elemene is a mixture isolated from Curcumae Rhizoma and is mainly composed of $\beta$-elemene ( $\beta$-ELE, Figure $1(a)$ ) [2]. Elemene exhibits various anti-cancer activities [2] and has already been approved as a new anti-cancer drug by the State Food and Drug Administration of China. However, Curcumae Rhizoma contains much less $\beta$-ELE than FUR [11]. Therefore, determination of whether or not FUR has anticancer activities similar to that of $\beta$-ELE could promote the use of the herb.

This study aims to (1) compare the anticancer effects of $\beta$-ELE and FUR in human lung cancer cells, (2) explore the possible anti-cancer mechanisms of FUR, and (3) detect 


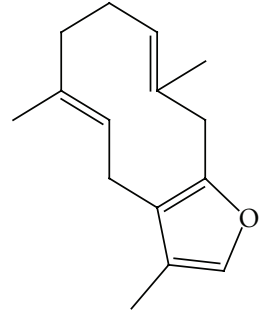

Furanodiene (FUR)

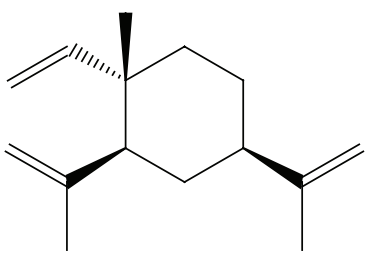

$\beta$-elemene $(\beta$-ELE)

(a)
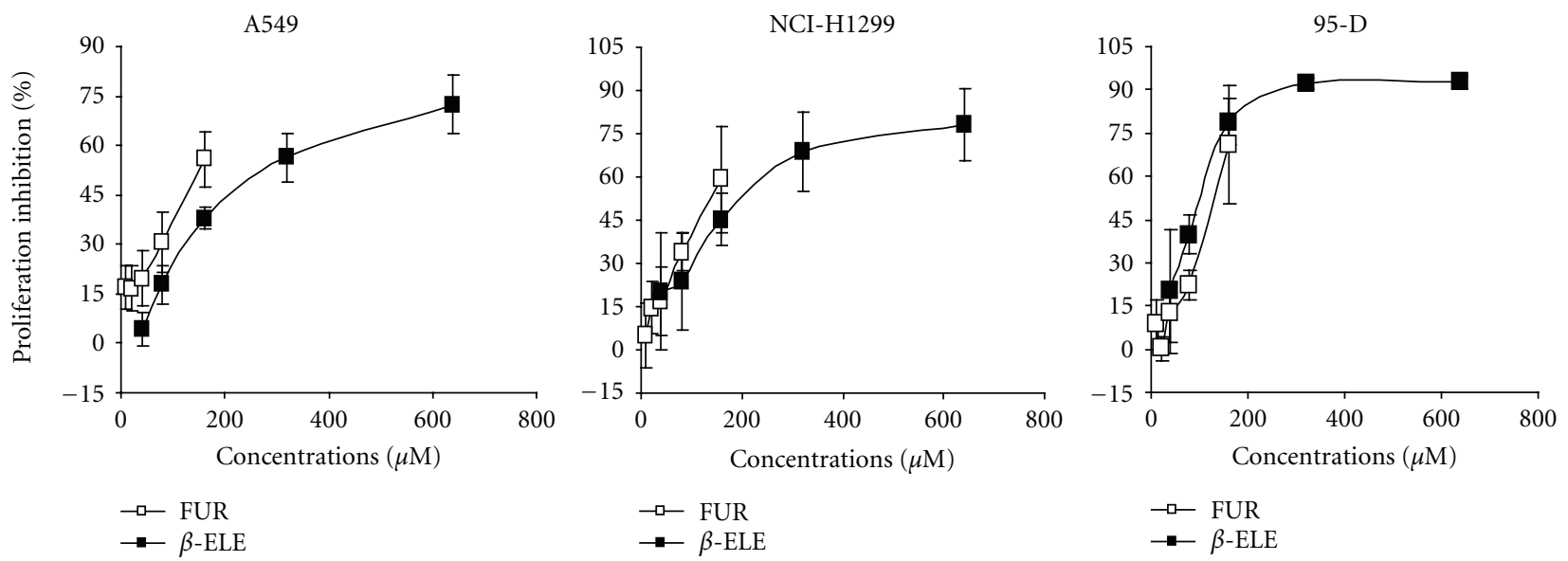

(b)

FIgURE 1: Comparison of FUR and $\beta$-ELE on lung cancer cell proliferation. (a) Chemical structure of FUR and $\beta$-ELE, (b) cells were treated with FUR or $\beta$-ELE for $48 \mathrm{~h}$, after which cell proliferative inhibition was tested by MTT assay.

the combined therapeutic effects of FUR with common chemotherapeutic agents. We found that FUR exhibits anticancer activities stronger than or similar to those of $\beta$-ELE in three tested lung cancer cell lines and that it retards colony formation and triggers endoplasmic reticulum stress (ER stress) in A549 and 95-D cells. The effects of the combined treatment of FUR and doxorubicin (DOX) or paclitaxel (TAX) were also investigated.

\section{Materials and Methods}

2.1. Materials. FUR was separated and purified from the essential oil of Curcuma wenyujin by silica-gel-column separation [12] and the purity of FUR $(>96 \%)$ was tested by HPLC-DAD [11]. $\beta$-ELE was purchased from WAKO (Japan). The stock concentration of $\beta$-ELE and FUR was $320 \mathrm{mM}$ and $160 \mathrm{mM}$, respectively. DOX and TAX were obtained from Sigma (St. Louis, MO, USA) and the stock concentration was $4 \mathrm{mM}$ and $1 \mathrm{mM}$, respectively. 3-[4,5-Dimethyl-2-thiazolyl]-2,5-diphenyl tetrazolium bromide (MTT) was purchased from Amersham (Sweden).

2.2. Cell Culture. The human lung cancer cell lines A549 (ATCC, Rockville, MD, USA), NCI-H1299 (ATCC), and 95D (The Cell Bank of Type Culture Collection of Chinese Academy of Sciences, Shanghai, China) were cultured in
RPMI 1640 medium supplemented with 10\% fetal bovine serum (GIBCO BRL, Carlsbad, CA, USA), $100 \mathrm{U} / \mathrm{mL}$ penicillin, and $100 \mathrm{mg} / \mathrm{mL}$ streptomycin and were grown in an incubator with $5 \% \mathrm{CO}_{2}$ at $37^{\circ} \mathrm{C}$.

2.3. MTT Assay. Exponentially growing A549, NCI-H1299, and 95-D cells were planted into 96-well plates, and then treated with serial concentrations of FUR or $\beta$-ELE for 48 $\mathrm{h}$ after adhesion. For the combinational treatment, FUR, DOX, and TAX were simultaneously added into the wells and treated for $48 \mathrm{~h}$ according to the combined treatment design of the experiment. Cell proliferation was determined by addition of $1 \mathrm{mg} / \mathrm{mL}$ MTT-containing medium for $4 \mathrm{~h}$, addition of $100 \mu \mathrm{L}$ DMSO to solubilize the formazan, and shaking for $10 \mathrm{~min}$ in the dark. The absorbance at $570 \mathrm{~nm}$ was recorded using a multilabel counter (Perkin Elmer, Singapore).

2.4. Observation of Morphological Changes. A549 and 95D cells were seeded into 6-well plates and treated with the indicated concentration of FUR for $24 \mathrm{~h}$. The cellular morphology was observed using an AxioCam HRC CCD camera (Carl Zeiss).

2.5. Colony Formation Assay. A549 and 95-D cells were treated with serial concentrations of FUR for $24 \mathrm{~h}$ (200 nM 
TAX was used as a positive control) and then suspended and reseeded into 6-well plates at a density of 200 cells per well (A549) or 1000 cells per well (95-D) after treatment. Cells were subsequently fixed using $4 \%$ paraformaldehyde and stained with crystal violet staining solution (Beyotime Institute of Biotechnology, China) after one-week incubation. Typical images were captured using an ordinary NIKON camera.

2.6. Real-Time PCR. A549 and 95-D cells were treated with $60 \mu \mathrm{M}$ and $80 \mu \mathrm{M}$ FUR for $12 \mathrm{~h}$ and the total RNA was extracted using the RNeasy mini kit (Qiagen, 74106) according to the manufacturer's protocol. The primer sequences were as follows: 5 -TCCTATGTCGCCTTCACT$3^{\prime}$ (forward), 5'-ACAGACGGGTCATTCCAC-3' (reverse) for binding immunoglobulin protein (BIP); $5^{\prime}$-CTGACCAGGGAAGTAGAGG-3' (forward)， $5^{\prime}$-TGCGTATGTGGGATTGAG-3' (reverse) for C/EBP homologous protein (CHOP); 5' -CCATGGAGAAGGCTGGGG-3' (forward); $5^{\prime}$ CAAATGTGTCATGGATGACC-3' (reverse) for GAPDH. One milligram of total RNA was reverse transcribed using the high capacity cDNA reverse transcription kit with RNase inhibitor (Invitrogen, 4374966) and the cDNA template was amplified by PCR using the Fast SYBR Green Master Mix (Applied biosystems, 4385616). Thermal cycling was programmed according to the manufacturer's protocol. Gene expression was assessed by $\Delta \Delta \mathrm{Ct}$ method and mRNA levels of $\mathrm{BIP}$ and $\mathrm{CHOP}$ were normalized to those of GAPDH internal standard.

2.7. Western Blot Analysis. Cells were lysed in the lysis buffer, and the proteins of the lysates were quantified using a BCA protein assay kit (Pierce, Rockford, IL, USA). About $30 \mu \mathrm{g}$ of total proteins was subjected to SDS-PAGE, transferred onto nitrocellulose membranes, and then blocked with 5\% nonfat milk in TBST ( $20 \mathrm{mM}$ Tris, $500 \mathrm{mM} \mathrm{NaCl}$, and $0.1 \%$ Tween-20) at room temperature for $2 \mathrm{~h}$ with rocking. The membranes were probed with specific primary antibodies against BIP and CHOP (Cell Signaling Technology, Beverly, MA, USA) overnight at $4^{\circ} \mathrm{C}$. After washing with TBST three times for $15 \mathrm{~min}$ each, the membranes were incubated with horseradish-peroxidase-conjugated secondary antibodies (Santa Cruz Biotechnology Inc.) in TBST at room temperature for $1 \mathrm{~h}$, and specific protein bands were visualized using an ECL advanced Western blot detection kit. Equal protein loading was verified through rehybridization of the membranes and reprobing with anti- $\beta$-actin antibody.

2.8. Immunocytochemical Labeling. A549 and 95-D cells were fixed with $4 \%$ formaldehyde in $\mathrm{PBS}$ at $37^{\circ} \mathrm{C}$ for $30 \mathrm{~min}$, washed with PBS, and then permeabilized with $0.5 \%$ Triton X-100 in PBS for $20 \mathrm{~min}$ at room temperature after FUR treatment. Cells were washed in a blocking solution containing 5\% BSA and $0.2 \%$ Triton X-100 and stored in the blocking solution at $4^{\circ} \mathrm{C}$ until labeling. Fixed cells were then incubated at $4^{\circ} \mathrm{C}$ with the specific antibody against $\mathrm{CHOP}$ in the blocking solution overnight. After three washes in the blocking solution, cells were incubated with goat anti-rabbit IgG in the blocking solution at $37^{\circ} \mathrm{C}$ for $1 \mathrm{~h}$. After three washes, cells were incubated for $10 \mathrm{~min}$ at room temperature with Hoechst 33342. Cells were then mounted in a $90 \%$ glycerol-PBS mixture after three washes with PBS, after which they were visualized and photographed with an IX81 fluorescent microscope (Olympus, Japan).

2.9. Statistical Analysis. Combination index (CI) is well accepted for quantifying drug synergism based on the multiple-drug effect equation of Chou-Talalay $[13,14]$. In the current study, CI values were tested for each concentration of FUR, DOX, or TAX, and the corresponding combination in cell proliferation assays using Calcusyn (Biosoft, Cambridge, United Kingdom). A CI lower than 0.9 indicates synergism, a CI of 0.9 to 1.10 indicates additive, and a CI higher than 1.10 indicates antagonism [15].

\section{Results}

3.1. Comparison of the Anticancer Effects of FUR and $\beta$ ELE on Lung Cancer Cells. $\beta$-ELE is an anti-cancer drug obtained from the same source as FUR [2, 11]; thus, the anti-proliferative activities of these two compounds in lung cancer cells were compared. Cells were treated with various concentrations of FUR or $\beta$-ELE for $48 \mathrm{~h}$, and both compounds inhibited cell proliferation in a concentrationdependent manner, as detected by MTT assay (Figure 1(b)). $\beta$-ELE showed weaker anti-proliferative activities compared with FUR in A549 and NIH-H1299 lung cancer cells (Figure 1(b)) and MCF-7 breast cancer cells (data not shown) but exhibited effects similar to FUR in 95-D cells (Figure 1(b)). This result indicates that FUR has similar or even better anti-cancer potential compared with $\beta$-ELE.

3.2. FUR Inhibits Colony Formation in Lung Cancer Cells. The colony formation potential of FUR on lung cancer A549 and 95-D cells after FUR treatment was determined. Cells were treated with serial concentrations of FUR for $24 \mathrm{~h}$, and morphological changes after FUR treatment were observed using phase-contrast microscopy (Figure 2(a)). FUR decreased the percentage of adherent cells in a concentration-dependent manner in both cell lines. In addition, the colony formation assay was used to further investigate the anti-cancer potential of FUR. $200 \mathrm{nM}$ TAX was used as a positive control. The number of colony foci decreased after treatment with $80 \mu \mathrm{M}$ FUR (Figure 2(b)) and TAX (data not shown) in both cell lines, consistent with the results of morphological changes (Figure 2(a)) and the MTT assay (Figure 1(b)).

\subsection{Induction of ER Stress after FUR Treatment in Lung Cancer Cells. The mechanisms by which FUR produces its anti-proliferative effects were also investigated in this study. Various compounds induce ER stress [16, 17], which can trigger a signal transduction network to induce cell proliferation inhibition. We therefore investigated whether or not FUR induces ER stress in lung cancer cells. Induction of BIP and CHOP in A549 and 95-D cells after $12 \mathrm{~h}$ of FUR treatment was first investigated because responses to ER}




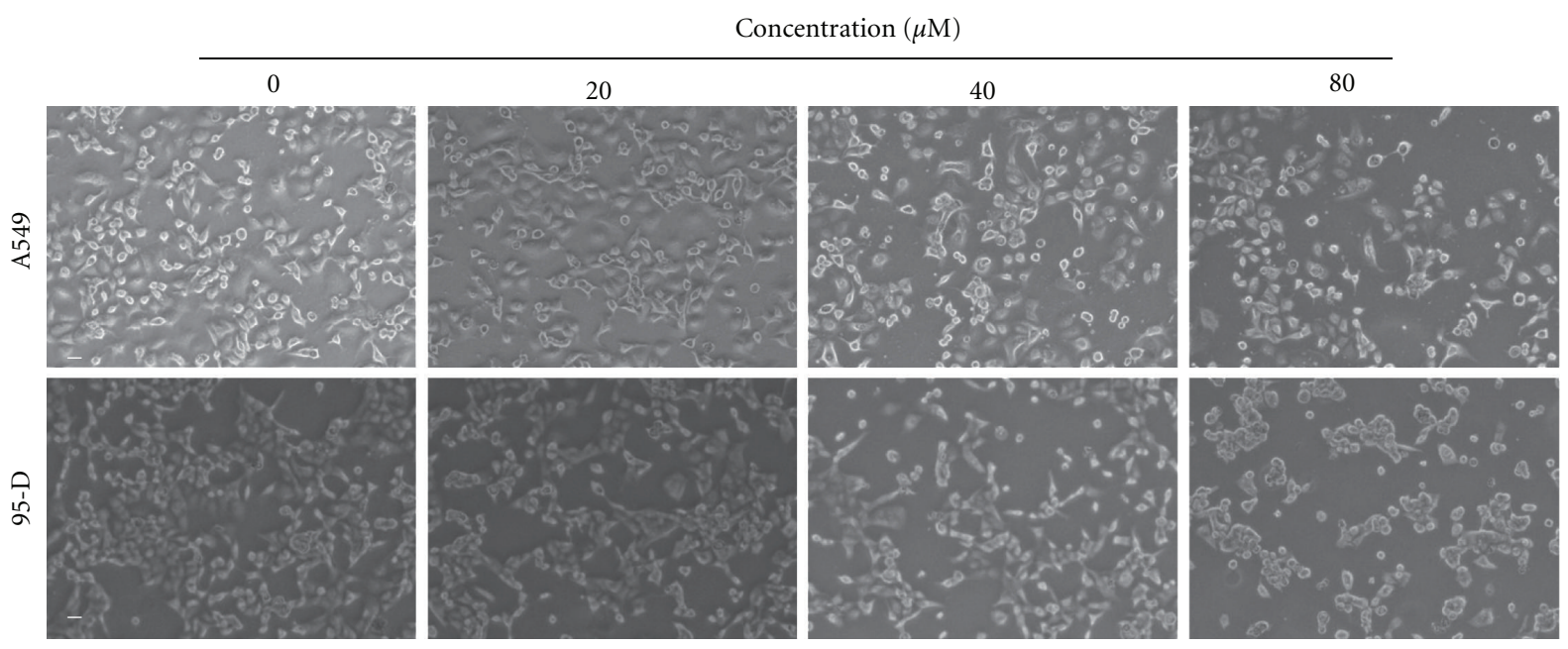

(a)

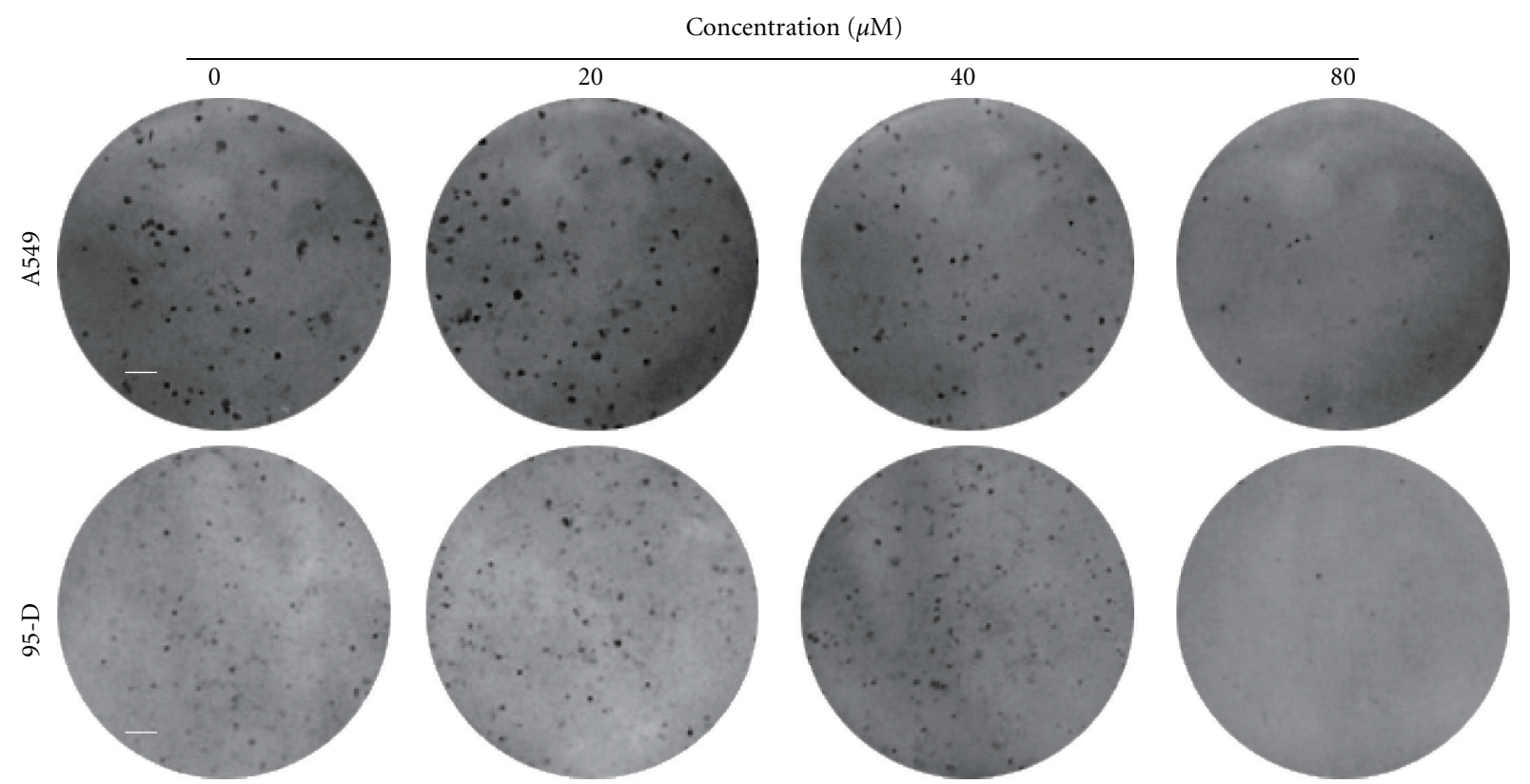

(b)

FIGURE 2: FUR inhibition on colony formation in lung cancer cells. (a) Cells were treated with various concentrations of FUR for 24 h, after which morphological changes were observed using an AxioCam HRC CCD phase contrast microscope. Bar: $20 \mu \mathrm{m}$. (b) Cells were treated with various concentrations of FUR for $24 \mathrm{~h}$. After treatment, cells were suspended and reseeded into 6-well plates at a density of 200 cells per well (A549) or 1000 cells per well (95-D) and then fixed and stained with 4\% PFA and crystal violet after one week. The representative images of colony formation assay were obtained. Bar: $2 \mathrm{~mm}$.

TABLE 1: CI values of FUR at concentrations applied in combination with DOX or TAX in different lung cancer cell lines.

\begin{tabular}{lcccccccc}
\hline \multirow{2}{*}{ DOX $(\mu \mathrm{M})$} & \multirow{2}{*}{ TAX $(\mu \mathrm{M})$} & \multirow{2}{*}{ FUR $(\mu \mathrm{M})$} & \multicolumn{2}{c}{$\mathrm{A} 549$} & \multicolumn{2}{c}{ NCI-H1299 } & \multicolumn{2}{c}{$95-\mathrm{D}$} \\
& & & $\mathrm{CI}_{\text {(DOX }+\mathrm{FUR})}$ & $\mathrm{CI}_{\text {(TAX }+\mathrm{FUR})}$ & $\mathrm{CI}_{\text {(DOX }+\mathrm{FUR})}$ & $\mathrm{CI}_{(\mathrm{TAX}+\mathrm{FUR})}$ & $\mathrm{CI}_{(\mathrm{DOX}+\mathrm{FUR})}$ & $\mathrm{CI}_{(\mathrm{TAX}+\mathrm{FUR})}$ \\
\hline 0.25 & 0.025 & 10 & $1.96 \pm 1.32$ & $0.15 \pm 0.07$ & $0.72 \pm 0.12$ & $0.17 \pm 0.14$ & $1.11 \pm 0.61$ & $0.11 \pm 0.11$ \\
0.5 & 0.05 & 20 & $1.35 \pm 0.52$ & $0.40 \pm 0.25$ & $1.48 \pm 0.15$ & $0.13 \pm 0.17$ & $1.63 \pm 0.49$ & $0.17 \pm 0.21$ \\
1 & 0.1 & 40 & $2.38 \pm 2.11$ & $0.61 \pm 0.16$ & $0.91 \pm 0.43$ & $0.16 \pm 0.15$ & $1.44 \pm 1.04$ & $0.25 \pm 0.19$ \\
2 & 0.2 & 80 & $0.63 \pm 0.12$ & $1.38 \pm 1.44$ & $0.40 \pm 0.02$ & $0.20 \pm 0.12$ & $0.42 \pm 0.15$ & $0.35 \pm 0.27$ \\
4 & 0.4 & 160 & $0.50 \pm 0.17$ & $0.66 \pm 0.04$ & $0.54 \pm 0.07$ & $0.18 \pm 0.13$ & $0.74 \pm 0.28$ & $0.35 \pm 0.29$ \\
\hline
\end{tabular}



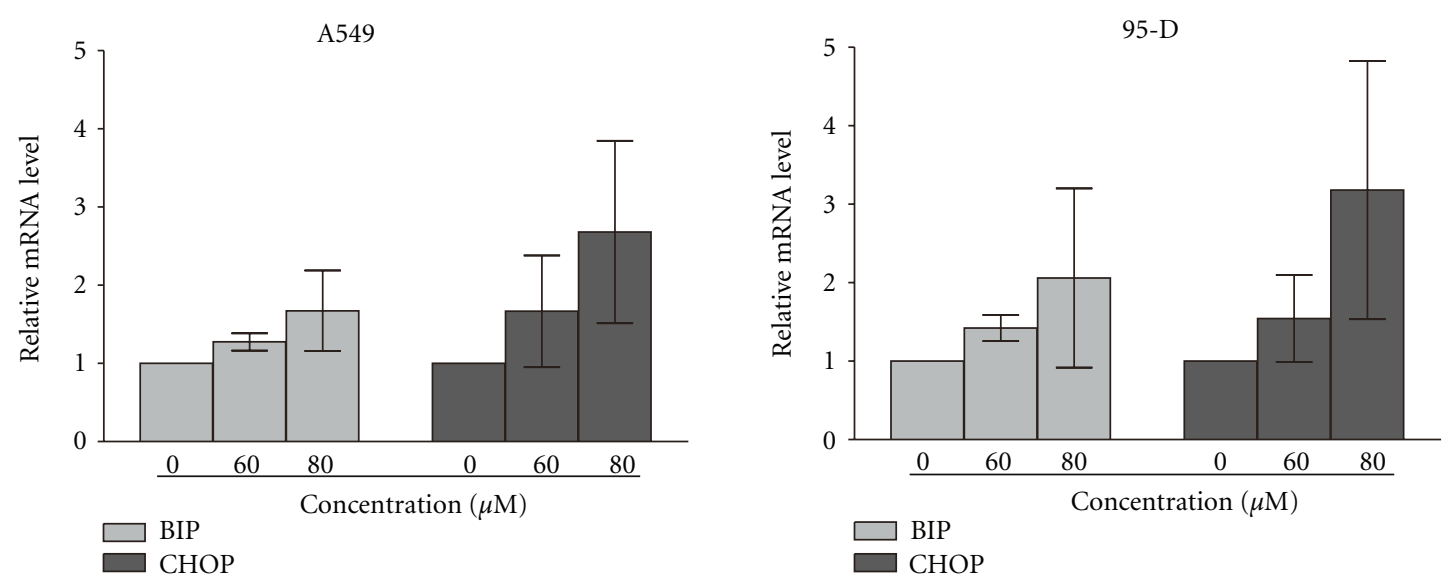

(a)
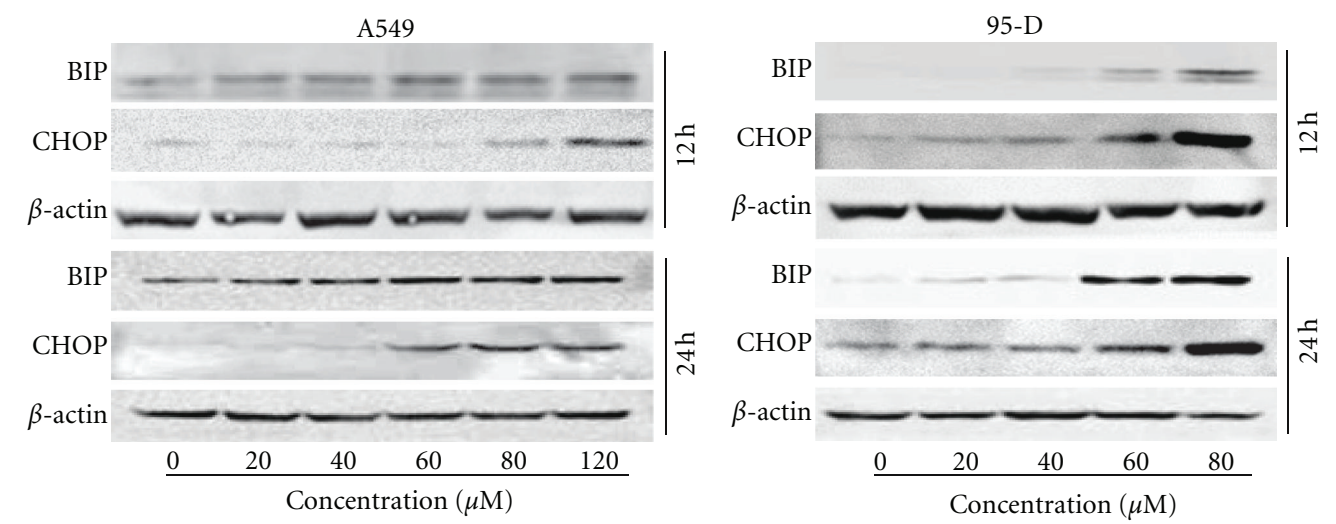

(b)

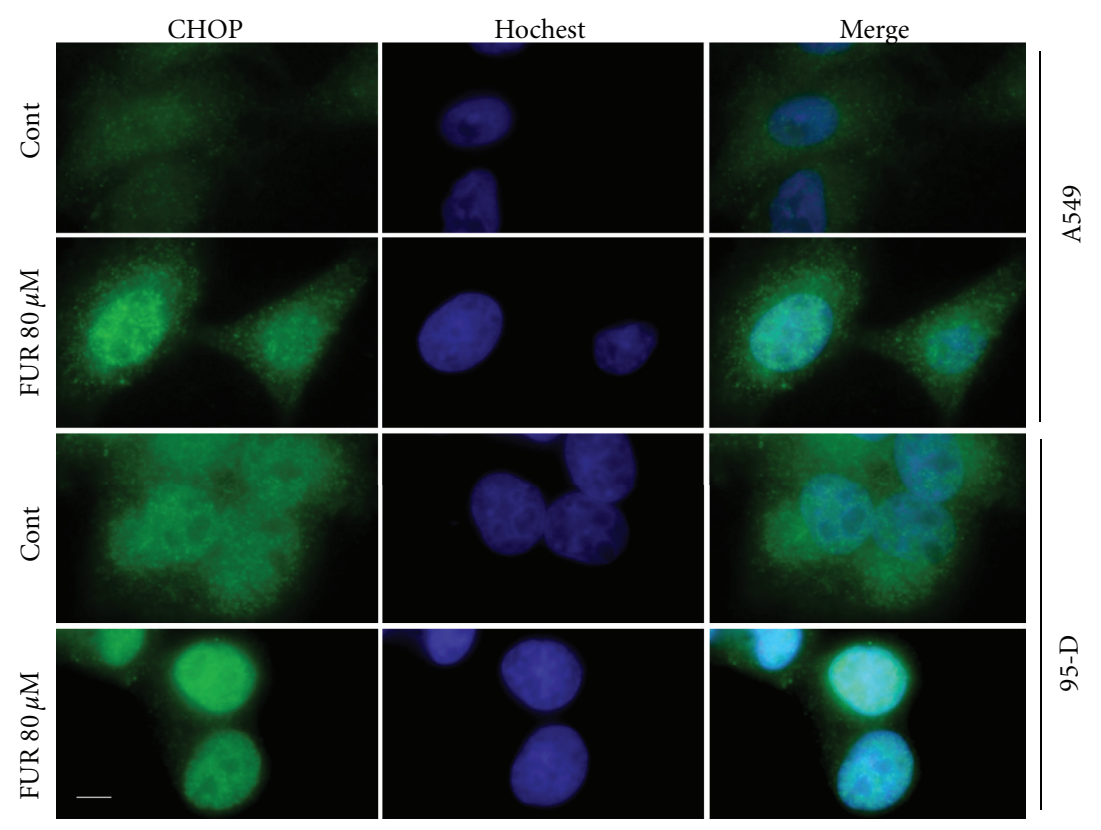

(c)

FIGURE 3: FUR induces ER stress in lung cancer cells. (a) Effect of FUR on the mRNA levels of BIP and CHOP in A549 and 95-D cells. Cells were treated with different concentrations of FUR for $12 \mathrm{~h}$, and mRNA levels were determined by RT-PCR. (b) Effect of FUR on the protein levels of BIP and CHOP in A549 and 95-D cells. Cells were treated with different concentrations of FUR for $12 \mathrm{~h}$ or $24 \mathrm{~h}$, and protein levels were determined by Western blot. (c) Immunocytochemical staining was conducted to detect the expression of CHOP in nuclei. Cells were treated with $80 \mu \mathrm{M}$ FUR for $12 \mathrm{~h}$ and the cells were fixed and stained with anti-CHOP antibody (green) and Hoechst 33342 (blue). Bar: $10 \mu \mathrm{m}$. 

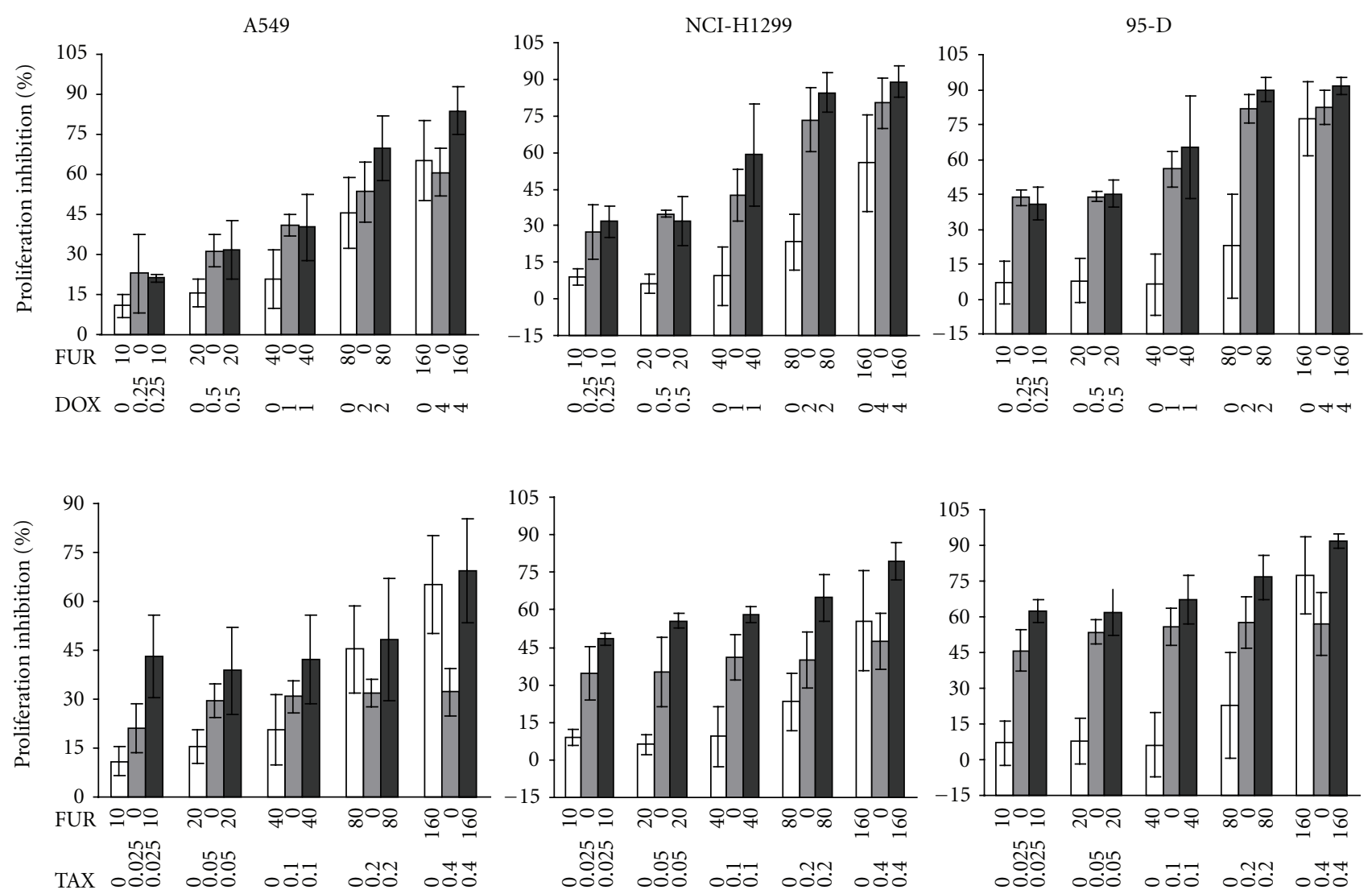

FIGURE 4: Combined cytotoxicity of FUR and DOX or FUR and TAX in three lung cancer cell lines. Cells in 96-well plates were treated with serial concentrations of FUR with or without the indicated DOX or TAX for $48 \mathrm{~h}$. Cell proliferative inhibition was tested using MTT assay.

stress are characterized in part by the increased transcription of BIP and induction of CHOP $[18,19]$. Figure $3(\mathrm{a})$ shows that FUR induced an increase in BIP and CHOP at the mRNA level in both cell lines. Induction of BIP and CHOP at the protein level in A549 and 95-D cells after $12 \mathrm{~h}$ and $24 \mathrm{~h}$ of FUR treatment was also confirmed (Figure 3(b)). Furthermore, the numbers of CHOP foci also increased after FUR treatment for $12 \mathrm{~h}$ in both cell lines (Figure $3(\mathrm{c})$ ) while CHOP foci were much more obvious in 95-D cells than those in A549 cells. In summary, FUR induces ER stress in A549 and 95-D lung cancer cells, which may contribute at least in part to its anti-cancer potential.

3.4. The Effects of FUR in Combination with DOX or TAX in Different Lung Cancer Cell Lines. The anti-proliferative effects of FUR were further determined through the combined treatment of FUR and DOX or FUR and TAX on A549, NCI-H1299, and 95-D human lung cancer cell lines. Cells in 96-well plates were treated with serial concentrations of FUR with or without the indicated DOX or TAX for $48 \mathrm{~h}$. The cell proliferative inhibition bars were shown in Figure 4. FUR, DOX, and TAX displayed cytotoxicity in a concentrationdependent manner against all three cancer cell lines. Much stronger anti-proliferation abilities were achieved after combinational treatment (except at low concentrations of DOX and FUR) compared with treatment with FUR, DOX or
TAX alone. CI values were calculated using Calcusyn at fixed ratio concentrations of FUR and DOX or FUR and TAX. Table 1 showed that FUR plus TAX presents obvious synergistic anti-cancer effects in NIH-H1299 and 95-D cells while this effect was weaker in A549 cells. FUR plus DOX also showed synergistic anti-cancer effects at high concentrations, whereas antagonism was observed at lower doses in all tested three cell lines (Table 1).

\section{Discussion}

In this study, FUR effectively inhibited cell proliferation in lung cancer cells compared with $\beta$-ELE, the main component of elemene. Curcumae Rhizoma contains much less $\beta$-ELE than FUR [11]; thus, FUR has potential application as an alternative for $\beta$-ELE. This study also further clarified the pharmacodynamic material basis for Curcumae Rhizoma.

Pharmacological alterations in glycosylation machinery, redox potential, or calcium levels disrupt normal ER protein biogenesis, resulting in ER stress [20]. As a protective mechanism, ER triggers unfolded protein responses to protect cells against ER stress. Therefore, cell death pathways are initiated when ER stress cannot be alleviated [20]. In the present study, FUR upregulated BIP and CHOP expression both in the mRNA and protein levels and accumulated $\mathrm{CHOP}$ in cell nuclei, all of which are markers of ER stress 
$[18,19]$, after $12 \mathrm{~h}$ of treatment. FUR-induced ER stress may trigger a series of cellular responses that lead to proliferative inhibition. However, the detailed mechanism for such an activity requires further study. In addition, we note that the ER stress trigged by FUR is not consistent in different cell lines, which may be due to the different cellular background.

$\mathrm{CI}$ is a well-accepted numerical value that provides a qualitative measure of the extent of drug interaction. In this study, CI was used to evaluate the combination effects of FUR and DOX or FUR and TAX on the proliferation of lung cancer A549, NCI-H1299, and 95-D cells. Our data showed that FUR remarkably potentiated TAX-imposed cytotoxicity, as revealed by CI values (Table 1), in NIH-H1299 and 95$\mathrm{D}$ cells. TAX is widely used in clinical settings to treat lung cancers $[21,22]$. Further research is recommended to investigate the possible mechanisms involved in such inhibition. It should be aware that the synergetic effects are dependent on the cell types as the synergetic effect is weaker in A549 cells.

In summary, FUR showed anti-proliferative effects similar to or even stronger than those of $\beta$-ELE and exhibited synergetic effects with TAX in some lung cancer cells. FURinduced ER stress may partially contribute to its anticancer potential, though several detailed mechanisms require further study. These findings indicate that FUR may have the potential to be an anti-cancer natural compound, though there is still a long way to go.

\author{
Abbreviations \\ BIP: Binding immunoglobulin protein. \\ CHOP: C/EBPhomologous protein. \\ CI: $\quad$ Combination index. \\ DMSO: Dimethyl sulfoxide. \\ DOX: Doxorubicin. \\ $\beta$-ELE: $\beta$-elemene. \\ ER stress: Endoplasmic reticulum stress. \\ FUR: $\quad$ Furanodiene. \\ MTT: 3-[4,5-Dimethyl-2-thiazolyl]-2,5- \\ diphenyl tetrazolium \\ bromide. \\ TAX: Paclitaxel.
}

\section{Conflict of Interests}

The authors declare that they have no conflict of interests.

\section{Acknowledgments}

This study was supported by the Research Fund of University of Macau (UL016/09Y4/CMS/WYT01/ICMS and MYRG208(Y2-L4)-ICMS11-WYT) and the Macao Science and Technology Development Fund (045/2011/A, 077/2011/A3). The authors greatly thank Dr. Hong Zhu from Zhejiang University for the statistical analyses. The authors also extremely thank Mr. Zhang-Feng Zhong, Mr. ShengPeng Wang, Ms. Wen Tan, Ms. Jiao-Lin Bao, and Ms. LanZhen Meng, all from University of Macau for their technical help.

\section{References}

[1] C. Gridelli, A. Rossi, and P. Maione, "2010 Consensus on Lung Cancer, new clinical recommendations and current status of biomarker assessment-first-line therapy," European Journal of Cancer, vol. 47, supplement 3, pp. S248-S257, 2011.

[2] W. Tan, J. Lu, M. Huang et al., "Anti-cancer natural products isolated from chinese medicinal herbs," Chinese Medicine, vol. 6, no. 1, p. 27, 2011.

[3] Y. Wang, J. J. Lu, L. He, and Q. Yu, “Triptolide (TPL) inhibits global transcription by inducing proteasome-dependent degradation of RNA polymerase II (Pol II)," PLoS One, vol. 6, no. 9, Article ID e23993, 2011.

[4] H. Zhu, X. W. Liu, T. Y. Cai et al., "Celastrol acts as a potent antimetastatic agent targeting $\beta 1$ integrin and inhibiting cellextracellular matrix adhesion, in part via the p38 mitogenactivated protein kinase pathway," Journal of Pharmacology and Experimental Therapeutics, vol. 334, no. 2, pp. 489-499, 2010.

[5] J. J. Lu, Y. J. Cai, and J. Ding, "The short-time treatment with curcumin sufficiently decreases cell viability, induces apoptosis and copper enhances these effects in multidrugresistant K562/A02 cells," Molecular and Cellular Biochemistry, vol. 360, no. 1-2, pp. 253-260, 2012.

[6] G. S. Wu, J. J. Lu, J. J. Guo et al., "Ganoderic acid DM, a natural triterpenoid, induces DNA damage, G1 cell cycle arrest and apoptosis in human breast cancer cells," Fitoterapia, vol. 83, no. 2, pp. 408-414, 2012.

[7] Q. Xia, K. J. Zhao, Z. G. Huang et al., "Molecular genetic and chemical assessment of rhizoma Curcumae in China," Journal of Agricultural and Food Chemistry, vol. 53, no. 15, pp. 60196026, 2005.

[8] X. Y. Sun, Y. P. Zheng, D. H. Lin, H. Zhang, F. Zhao, and C. S. Yuan, "Potential anti-cancer activities of furanodiene, a sesquiterpene from Curcuma wenyujin," American Journal of Chinese Medicine, vol. 37, no. 3, pp. 589-596, 2009.

[9] E. Ma, X. Wang, Y. Li et al., "Induction of apoptosis by furanodiene in HL60 leukemia cells through activation of TNFR1 signaling pathway," Cancer Letters, vol. 271, no. 1, pp. 158-166, 2008.

[10] Y. Xiao, F. Q. Yang, S. P. Li et al., "Furanodiene induces G2/M cell cycle arrest and apoptosis through MAPK signaling and mitochondria-caspase pathway in human hepatocellular carcinoma cells," Cancer Biology and Therapy, vol. 6, no. 7, pp. 1044-1050, 2007.

[11] F. Q. Yang, Y. T. Wang, and S. P. Li, "Simultaneous determination of 11 characteristic components in three species of Curcuma rhizomes using pressurized liquid extraction and high-performance liquid chromatography," Journal of Chromatography A, vol. 1134, no. 1-2, pp. 226-231, 2006.

[12] F. Q. Yang, S. P. Li, Y. Chen et al., "Identification and quantitation of eleven sesquiterpenes in three species of Curcuma rhizomes by pressurized liquid extraction and gas chromatography-mass spectrometry," Journal of Pharmaceutical and Biomedical Analysis, vol. 39, no. 3-4, pp. 552-558, 2005.

[13] T. C. Chou and P. Talalay, "Generalized equations for the analysis of inhibitions of Michaelis-Menten and higher-order kinetic systems with two or more mutually exclusive and 
nonexclusive inhibitors," European Journal of Biochemistry, vol. 115, no. 1, pp. 207-216, 1981.

[14] T. C. Chou and P. Talalay, "Quantitative analysis of doseeffect relationships: the combined effects of multiple drugs or enzyme inhibitors," Advances in Enzyme Regulation, vol. 22, pp. 27-55, 1984.

[15] H. Zhu, W. J. Ding, R. Wu et al., "Synergistic anti-cancer activity by the combination of TRAIL/APO-2L and celastrol," Cancer Investigation, vol. 28, no. 1, pp. 23-32, 2010.

[16] J. J. Lu, S. M. Chen, X. W. Zhang, J. Ding, and L. H. Meng, "The anti-cancer activity of dihydroartemisinin is associated with induction of iron-dependent endoplasmic reticulum stress in colorectal carcinoma HCT116 cells," Investigational New Drugs, vol. 29, no. 6, pp. 1276-1283, 2011.

[17] H. O. Pae, S. O. Jeong, G. S. Jeong et al., "Curcumin induces pro-apoptotic endoplasmic reticulum stress in human leukemia HL-60 cells," Biochemical and Biophysical Research Communications, vol. 353, no. 4, pp. 1040-1045, 2007.

[18] X. Z. Wang, B. Lawson, J. W. Brewer et al., "Signals from the stressed endoplasmic reticulum induce C/EBP-homologous protein (CHOP/GADD153)," Molecular and Cellular Biology, vol. 16, no. 8, pp. 4273-4280, 1996.

[19] A. S. Lee, "Mammalian stress response: induction of the glucose-regulated protein family," Current Opinion in Cell Biology, vol. 4, no. 2, pp. 267-273, 1992.

[20] I. Kim, W. Xu, and J. C. Reed, "Cell death and endoplasmic reticulum stress: disease relevance and therapeutic opportunities," Nature Reviews Drug Discovery, vol. 7, no. 12, pp. 10131030, 2008.

[21] X. Y. Wang and Y. L. Zhao, "Clinical efficacy and adverse effects of taxol plus carboplatin or gemcitabine plus carboplatin in patients with advanced non-small-cell lung carcinoma," Zhonghua Yi Xue Za Zhi, vol. 90, no. 47, pp. 3362-3364, 2010.

[22] M. Li, H. Huang, J. Tan, and D. Lin, "A randomized clinical trial on taxol plus oxaliplatin versus taxol plus cisplatin as first-line treatment in advanced non-small cell lung cancer," Zhongguo Fei Ai Za Zhi, vol. 9, no. 5, pp. 452-454, 2006. 


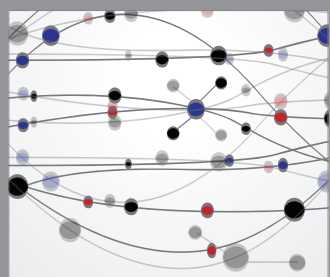

The Scientific World Journal
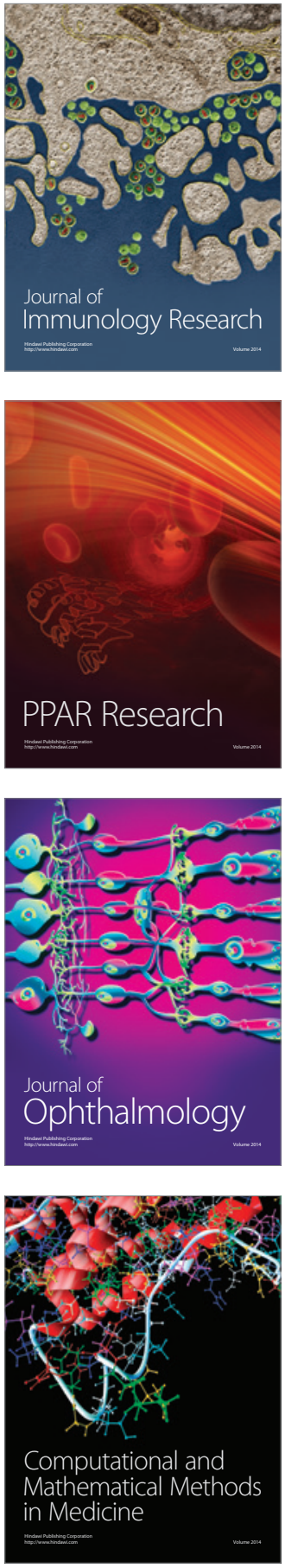

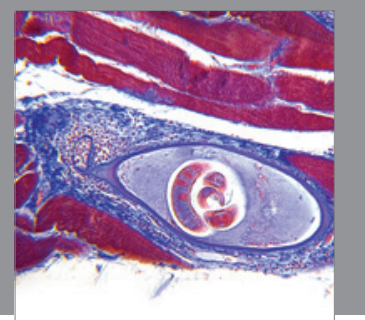

Gastroenterology

Research and Practice
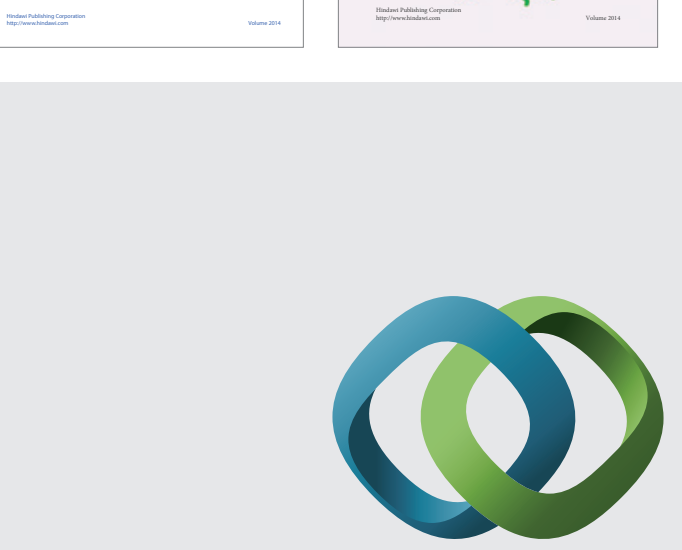

\section{Hindawi}

Submit your manuscripts at

http://www.hindawi.com
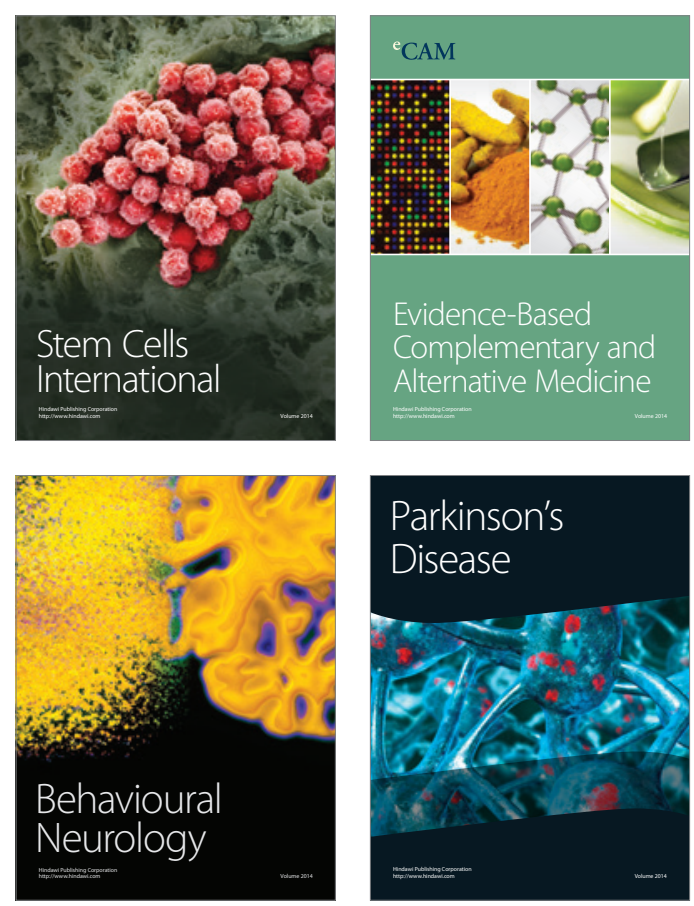

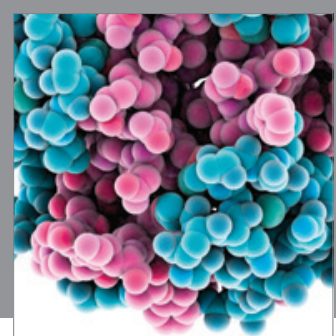

Journal of
Diabetes Research

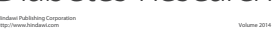

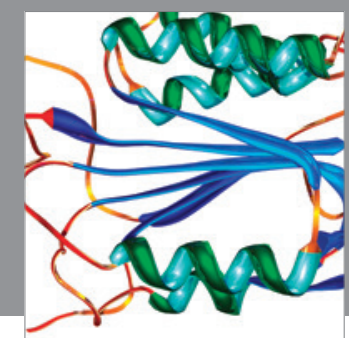

Disease Markers
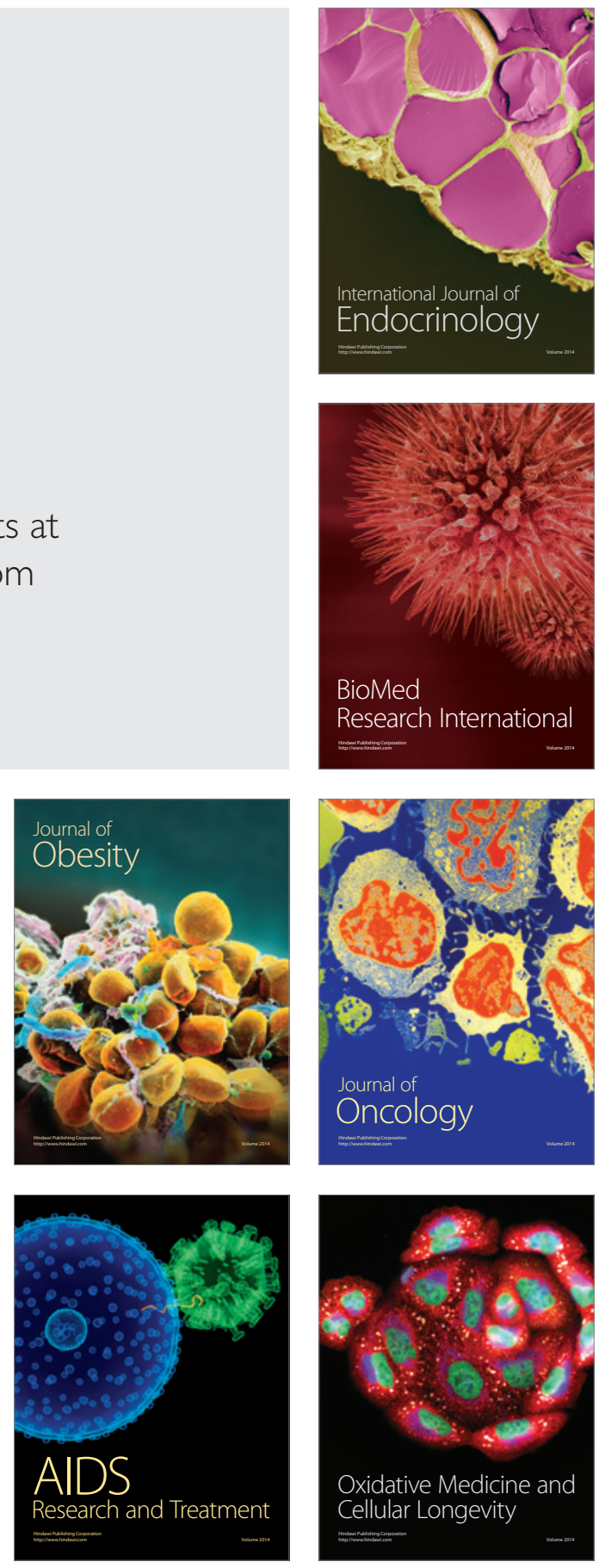